\title{
Reflecting properties in continuous images of small weight
}

\author{
Ofelia T. Alas $^{1}$ • Lucia R. Junqueira ${ }^{1}$. \\ Richard G. Wilson ${ }^{2}$
}

Received: 4 September 2015 / Revised: 2 December 2015 / Accepted: 20 December 2015 /

Published online: 11 February 2016

(C) Springer International Publishing AG 2016

\begin{abstract}
A topological property $\mathcal{P}$ is reflected in continuous images of weight at most $\omega_{1}$ if a space $X$ has $\mathcal{P}$ whenever every continuous image of $X$ of weight at most $\omega_{1}$ has $\mathcal{P}$. When $X$ is a generalized ordered space, we consider a number of topological properties including feeble Lindelöfness and $\kappa$-monolithicity. In the final section we study small images of pseudocompact and countably compact spaces; we give a condition on continuous images of a pseudocompact space in order that it be compact and show that it is consistently true that a countably compact space of countable projective tightness is countably tight.
\end{abstract}

Keywords GO-space · Pseudocompact space · Countably compact space . $\omega$-bounded space $\cdot$ Pseudocharacter $\cdot$ Countable projective tightness

Research is supported by Fundação de Amparo a Pesquisa do Estado de São Paulo, Grant 2014/26880-0 (Brasil), and CONACyT Grant CB-2012-01-178103 (México). Richard G. Wilson wishes to thank the Instituto de Matemática e Estatística da Universidade de São Paulo for their hospitality during the preparation of this paper.

$\bowtie \quad$ Lucia R. Junqueira

lucia@ime.usp.br

Ofelia T. Alas

alas@ime.usp.br

Richard G. Wilson

rgw@xanum.uam.mx

1 Instituto de Matemática e Estatística, Universidade de São Paulo, Caixa Postal 66281, São Paulo 05314-970, Brazil

2 Departamento de Matemáticas, Universidad Autónoma Metropolitana, Unidad Iztapalapa, Avenida San Rafael Atlixco, \# 186, Apartado Postal 55-532, 09340 Mexico, D.F., Mexico 
Mathematics Subject Classification $54 \mathrm{C} 05 \cdot 54 \mathrm{~F} 05 \cdot 54 \mathrm{D} 20 \cdot 54 \mathrm{~A} 25$

\section{Introduction and terminology}

Following the notation and terminology of $[10,11,13]$, we say that a topological property $\mathcal{P}$ is reflectable in continuous images of weight $\kappa$ in a class of topological spaces $\mathcal{C}$ if a space $X \in \mathcal{C}$ has the property $\mathcal{P}$ whenever every continuous image of $X$ of weight at most $\kappa$ has $\mathcal{P}$. This paper is a sequel to [13] in which $\mathcal{C}$ is the class of generalized ordered (or GO-) spaces, that is, the class of all subspaces of ordered spaces. It was shown in [13] that in GO-spaces, unlike in the class of all Tychonoff spaces, density, network weight and tightness are all reflected in small continuous images. Among the properties considered here are that of being $\kappa$-monolithic, having countable extent, pseudocompactness and countable compactness. We answer a number of questions posed in [13].

Recall that for an arbitrary cardinal $\kappa$, a space $(X, \tau)$ has extent equal to $\kappa$, written $\mathrm{e}(X)=\kappa$, if $\sup \{|D|: D \subseteq X$ is closed and discrete $\}=\kappa$; a space $X$ is $\kappa$-monolithic (respectively, strongly $\kappa$-monolithic) if for each $A \subseteq X$ with $|A| \leqslant \kappa$, we have $\mathrm{nw}(\operatorname{cl}(A)) \leqslant \kappa($ respectively, $\mathrm{w}(\operatorname{cl}(A)) \leqslant \kappa)($ see [12, Section 1.2]). A space is monolithic if it is $\kappa$-monolithic for each $\kappa$. Since for each subset $S$ of a GO-space $\mathrm{w}(S)=\mathrm{nw}(S)$, it follows that monolithicity and strong monolithicity coincide in this class. A space $X$ is strongly collectionwise Hausdorff if every closed discrete subset of $X$ can be separated by a discrete family of open sets.

If $X$ is a GO-space, then $p \in X$ is a left (respectively, right) pseudogap if $[p, \rightarrow$ ) is open and $p$ has no immediate predecessor (respectively, $(\leftarrow, p]$ is open and $p$ has no immediate successor). A point $p \in X$ is a pseudogap of $X$ if it is either a right or a left pseudogap. A jump of $X$ is a pair of consecutive elements of $X$. Let $X^{*}$ denote the (minimal) Dedekind completion of $X$ (obtained by adding one point in each gap and in each pseudogap); intervals in $X^{*}$ are denoted by $(a, c)^{*}$, while suprema and infima in $X^{*}$ are denoted by sup* and inf* and closures by $\mathrm{cl}^{*}$.

The remaining notation and terminology is standard and follows $[3,6]$; basic information regarding GO-spaces can be found in [7]. Throughout the paper, all spaces are assumed to be Tychonoff.

\section{Continuous images of GO-spaces}

In the sequel we shall use the following known result a number of times; the proof is left to the reader.

Lemma 2.1 If $X$ is a Tychonoff space and $a, b \in X$, then the quotient space $Y$ obtained from $X$ by identifying $a$ and $b$ is Tychonoff.

The following theorem answers [13, Question 4.7]; the much simplified proof was communicated to us by the referee.

Theorem 2.2 For any infinite cardinal $\kappa$, if every continuous image of a GO space $X$ of weight at most $\kappa^{+}$is $\kappa$-monolithic, then $X$ is $\kappa$-monolithic. 
Proof Suppose that the set $D \subseteq X$ is such that with $|D| \leqslant \kappa$. Then $Y=\operatorname{cl}(D)$ is also a GO space and we need to prove that nw $(Y) \leqslant \kappa$. Suppose that $f: Y \rightarrow Z$ is a continuous surjective map and $\mathrm{w}(Z) \leqslant \kappa^{+}$; there is no loss of generality if we assume that $Z \subseteq[0,1]^{\kappa^{+}}$.

The set $Y$ is closed in $X$ and so it follows from the normality of $X$ that there exists a continuous map $g: X \rightarrow[0,1]^{\kappa^{+}}$such that $g \mid Y=f$; then $Z^{\prime}=g[X] \supseteq Z$. The space $Z^{\prime}$ must be $\kappa$-monolithic being a continuous image of $X$ of weight at most $\kappa^{+}$, and so since $\mathrm{d}(Z) \leqslant \mathrm{d}(Y) \leqslant \kappa$ it follows that nw $(Z) \leqslant \kappa$. Therefore we have proved that every continuous image of $Y$ of weight not exceeding $\kappa^{+}$must have network weight at most $\kappa$. Since $Y$ is a GO space, we can apply [13, Theorem 3.11] to see that $\mathrm{w}(Y) \leqslant \kappa$ and hence $\mathrm{nw}(Y) \leqslant \kappa$, that is, $X$ is $\kappa$-monolithic.

Recall that a space $X$ is feebly $\kappa$-Lindelöf if every locally finite family of non-empty open sets is of cardinality at most $\kappa$. In case $\kappa=\omega$, then $X$ is said to be a feebly Lindelöf space. It is well known that if $X$ is a regular space, then for each $\kappa$, being feebly $\kappa$ Lindelöf is equivalent to the discrete $\kappa$ chain condition, that is, every discrete family of non-empty open sets is of cardinality at most $\kappa$.

[11, Example 2.4] shows that in the class of Tychonoff spaces having countable extent does not reflect in continuous images of weight $\omega_{1}$. However, the situation is different in the class of GO-spaces. If $D=\left\{d_{\alpha}: \alpha \in \kappa\right\}$ is a closed discrete subset of a GO-space $X$, then by collectionwise normality of $X$ there is a discrete family of open sets $\mathcal{U}=\left\{U_{\alpha}: \alpha \in \kappa\right\}$ such that $d_{\alpha} \in U_{\alpha}$ for each $\alpha \in \kappa$. It follows immediately that a GO-space $X$ is feebly $\kappa$-Lindelöf if and only if $\mathrm{e}(X) \leqslant \kappa$.

Theorem 2.3 The property of being feebly $\kappa$-Lindelöf is reflected in continuous images of weight $\kappa^{+}$.

Proof Suppose that $\left\{F_{\alpha}: \alpha \in \kappa^{+}\right\}$is a discrete family of non-empty open sets in $X$ and for each $\alpha \in \kappa^{+}$, pick $x_{\alpha} \in F_{\alpha}$. A simple well known argument can be used to show that there is a continuous function $f: X \rightarrow J\left(\kappa^{+}\right)$, the hedgehog with $\kappa^{+}$ spines (see [3, Example 4.1.5]) with the property that $f$ maps $x_{\alpha}$ to the end point of the $\alpha$ 'th spine and $X \backslash \bigcup\left\{F_{\alpha}: \alpha \in \kappa^{+}\right\}$to the vertex. Thus $f[X]$ has weight $\kappa^{+}$and is not feebly $\kappa$-Lindelöf.

The following result is now an immediate consequence of the lemma and the fact that if a surjective map $g: X \rightarrow Y$ is continuous and $\mathrm{e}(Y) \geqslant \kappa$, then $\mathrm{e}(X) \geqslant \kappa$ as well.

Theorem 2.4 If $X$ is a strongly collectionwise Hausdorff space, then $\mathrm{e}(X) \leqslant \kappa$ if and only if every continuous image of $X$ of weight at most $\kappa^{+}$has extent at most $\kappa$.

In the class of GO-spaces even more is true, and the following result seems to be of interest in its own right.

Theorem 2.5 Suppose that $\kappa$ is an infinite cardinal; if $X$ is a GO-space, and $\mathrm{e}(X) \geqslant$ $\kappa^{+}$, then $X$ can be continuously mapped onto the discrete space of cardinality $\kappa^{+}$.

Proof Suppose that $\mathrm{e}(X) \geqslant \kappa^{+}$and that $D$ is a closed discrete subset of $(X,<)$ of cardinality $\kappa^{+}$. Note that if $J$ is a countably compact interval in $(X,<)$, then $J \cap D$ is 
finite and hence any Dedekind complete subinterval of $X$ can contain at most countably many elements of $D$. We define an equivalence relation $\sim$ on $D$ as follows:

$$
\begin{aligned}
& d \sim d^{\prime} \Longleftrightarrow \quad \begin{array}{l}
\text { there is no element } p \in X^{*} \backslash X \\
\text { such that } p \in\left(d, d^{\prime}\right)^{*} \text { or } p \in\left(d^{\prime}, d\right)^{*} .
\end{array}
\end{aligned}
$$

Clearly then, there are $\kappa^{+}$-many equivalence classes, each of which is countable and without loss of generality, by passing to a subset of $D$, we may assume each equivalence class contains a unique element of $D$; in other words, between any two elements of $D$ there is a gap or a pseudogap. Well order $D$ as $\left\{d_{\alpha}: \alpha \in \kappa^{+}\right\}$and consider $d_{0} \in D$. Let $c_{0}=\sup ^{*}\left\{d \in D: d<d_{0}\right\}$ and $e_{0}=\inf ^{*}\left\{d \in D: d>d_{0}\right\}$. Since $D$ is closed and discrete in $X$, if $z \in\left\{c_{0}, d_{0}\right\}$, then either

$$
z \in X^{*} \backslash X \quad \text { or } \quad z \in D \backslash\left\{d_{0}\right\}
$$

If $c_{0} \in X^{*} \backslash X$, then put $p_{0}=c_{0}$ and if $c_{0} \in D$, then pick an element $p_{0} \in X^{*} \backslash X$ such that $c_{0}<p_{0}<d_{0}$. Similarly we may find $q_{0} \in X^{*} \backslash X$ such that $d_{0}<q_{0} \leqslant e_{0}$ and the resulting clopen subinterval $I_{0}=\left(p_{0}, q_{0}\right)^{*} \cap X$ of $X$ is such that $I_{0} \cap D=\left\{d_{0}\right\}$. Suppose now that $\alpha<\kappa^{+}$and that we have constructed disjoint clopen intervals $I_{\beta}=\left(p_{\beta}, q_{\beta}\right)^{*} \cap X$, where $p_{\beta}, q_{\beta} \in X^{*} v \backslash X$ and $I_{\beta} \cap D=\left\{d_{\beta}\right\}$ for each $\beta<\alpha$; consider $d_{\alpha} \in D$. It is now easy to see that since $D$ is closed and discrete, it follows that $d_{\alpha} \notin \operatorname{cl}\left(\bigcup\left\{I_{\beta}: \beta<\alpha\right\}\right)$. Thus

$$
\begin{aligned}
c_{\alpha} & =\sup ^{*}\left\{d_{\beta}: \beta<\alpha \text { and } d_{\beta}<d_{\alpha}\right\}<d_{\alpha} \\
& <\inf ^{*}\left\{d_{\beta}: \beta<\alpha \text { and } d_{\beta}>d_{\alpha}\right\}=e_{\alpha} .
\end{aligned}
$$

As before, if $c_{\alpha} \in X^{*} \backslash X$, then clearly $c_{\alpha} \geqslant q_{\beta}$ for all $q_{\beta}$ such that $\beta<\alpha$ and $d_{\beta}<d_{\alpha}$ and we put $p_{\alpha}=c_{\alpha}$. If on the other hand, $c_{\alpha} \in D \backslash\left\{d_{\alpha}\right\}$, then there is $p_{\alpha} \in X^{*} \backslash X$ such that $c_{\alpha}<p_{\alpha}<d_{\alpha}$. A similar argument can be used to find $q_{\alpha} \in X^{*} \backslash X$ such that $I_{\alpha}=\left(p_{\alpha}, q_{\alpha}\right)^{*} \cap X$ is a clopen interval in $X$ disjoint from all the intervals $I_{\beta}$ and $I_{\alpha} \cap D=\left\{d_{\alpha}\right\}$.

Now for each element $d \in D$ suppose that we have chosen $p_{d}, q_{d} \in X^{*} \backslash X$ such that $\left(p_{d}, q_{d}\right)^{*} \cap D=\{d\}$ and the intervals $I_{d}=\left(p_{d}, q_{d}\right)^{*} \cap X$ are mutually disjoint; we claim that the family $\left\{I_{d}: d \in D\right\}$ is discrete. To prove the claim, notice that since $D$ is closed and discrete and $X$ is collectionwise normal, there is a discrete family of open sets $\mathcal{U}=\left\{U_{d}: d \in D\right\}$ such that $d \in U_{d}$ and without loss of generality we may assume that $U_{d} \subseteq I_{d}$. Suppose, contrary to our claim, that $x \in X$ is an accumulation point of the family $\left\{I_{d}: d \in D\right\}$; then each open interval $J$ in $X$ with $x \in J$ meets infinitely many of the sets $I_{d}$. However, this implies that $J$ contains infinitely many intervals $I_{d}$, and hence infinitely many intervals $U_{d}$; this contradicts the fact that $\mathcal{U}$ is a discrete family.

Since each interval $I_{d}$ is open and closed in $X$, it now follows that $\bigcup\left\{I_{d}: d \in D\right\}$ is also open and closed (in $X$ ) and we may define a map $f: X \rightarrow D$ by $f\left[I_{d}\right]=\{d\}$ and $f\left[X \backslash \bigcup\left\{I_{d}: d \in D\right\}\right]=\left\{d_{0}\right\}$, where $d_{0} \in D$; clearly, $f$ is a continuous map from $X$ onto the discrete space of cardinality $\kappa^{+}$. 
Following [13], we say that a space $X$ has countable projective tightness if every continuous image $Y$ of $X$ with $\mathrm{w}(Y) \leqslant \omega_{1}$ has countable tightness. It is easy to see that any hereditarily separable space has countable projective tightness. If $X$ is a space which is not feebly Lindelöf, then it contains a closed, discrete, $C$-embedded subset $A$ of cardinality $\omega_{1}$ which can be mapped continuously onto the one-point Lindelöfication of a discrete space of the same cardinality which we assume to be embedded in $\mathbb{R}^{\omega_{1}}$. As in [10, Lemma 3.3] this map can be extended continuously to $X$, showing that $X$ does not have countable projective tightness. The following result is an immediate consequence.

Proposition 2.6 If $X$ has countable projective tightness, then $X$ is feebly Lindelöf and if $X$ is normal, in particular, if $X$ is a GO-space, then $\mathrm{e}(X)=\omega$.

In [13] it was shown that a GO-space of countable projective tightness has countable tightness and Proposition 2.6 shows that it must have countable extent. However, even more is true.

Theorem 2.7 If $X$ is a GO-space which has countable projective tightness, then $X$ is a Lindelöf space.

Proof Note first that if there is some point $p \in X^{*} \backslash X$ such that $\mathrm{t}\left(p, X^{*}\right)>\omega$, then the quotient topology on $X$ obtained by identifying $p$ and a point $x \in X$ in the space $X \cup\{p\}$ is, by Lemma 2.1, a Tychonoff space and has uncountable tightness. It follows that every gap and pseudogap of $X$ must have countable coinitiality and countable cofinality; it now follows from [8, Theorem E], that $X$ is paracompact. Then, if $X$ is not Lindelöf, there must exist a locally finite uncountable open cover of the space $X$ and by choosing a point in each element of this cover we obtain an uncountable closed, discrete subset of $X$. This contradicts Proposition 2.6, and so $X$ is Lindelöf.

Since in the class of compact spaces, countable tightness is preserved under continuous images, the lexicographically ordered square $[0,1]^{2}$ shows that a LOTS of countable projective tightness need be neither separable nor hereditarily Lindelöf.

The question was posed in [13] as to whether, in the class of GO-spaces, $\sigma$-pseudocompactness (or equivalently, $\sigma$-countable compactness) is reflected in continuous images of weight $\omega_{1}$. We note here only that a $\sigma$-pseudocompact space is feebly Lindelöf, but the converse is false even in the class of first countable GO-spaces, as the space of irrationals illustrates.

\section{Small images of pseudocompact spaces}

If $X$ is a non-pseudocompact space, then it contains a closed, discrete, $C$-embedded, countably infinite subspace $D$. The set $D$ can be mapped continuously onto a countable dense subspace of $\mathbb{R}^{\omega_{1}}$ and then (as in [10, Lemma 3.3]) this function can be extended continuously to all of $X$; thus $X$ has a continuous image of weight at most $\omega_{1}$ which does not have any point of countable character. The next theorem considers the case in which $X$ is pseudocompact and answers positively [13, Question 4.4]. 
Theorem 3.1 A pseudocompact Tychonoff space $X$ has the property that every continuous image $Y$ of $X$ with $\mathrm{w}(Y) \leqslant \omega_{1}$ has countable pseudocharacter if and only if $X$ is compact and perfect.

Proof To simplify the terminology, let us say that a space $X$ has property $P$ if it is a pseudocompact Tychonoff space such that every continuous image $Y$ of $X$ with $\mathrm{w}(Y) \leqslant \omega_{1}$ has countable pseudocharacter.

It follows immediately from [13, Proposition 3.3 (b)] that if $X$ has property $P$, then it is perfect and so $\psi(X)=\omega$. Since both $X$ and $Y$ are pseudocompact, a folklore result which appears in a slightly stronger form as [1, Lemma 3.7] then implies that $\chi(X)=\chi(Y)=\omega$ also.

Suppose now that the space $X$ has property $P$ and $Y$ is a continuous image of $X$ of weight at most $\omega_{1}$ which is not compact. Then since $Y$ is pseudocompact, it is not realcompact, and so it can be embedded in $\mathbb{R}^{\omega_{1}}$ as a non-closed subspace $(Z, \tau)$. Furthermore, the closure of $Z$ in $\mathbb{R}^{\omega_{1}}$ is a realcompact, pseudocompact space and is therefore compact. Let $z \in Z, p \in \operatorname{cl}(Z) \backslash Z$ and consider the quotient space obtained by identifying $p$ and $z$ in the space $Z \cup\{p\}$; for convenience we denote this space by $\left(Z, \tau^{\prime}\right)$. Note that since $\chi(p, Z \cup\{p\}) \leqslant \mathrm{w}\left(\mathbb{R}^{\omega_{1}}\right)=\omega_{1}$ it follows that $\mathrm{w}\left(Z, \tau^{\prime}\right) \leqslant \omega_{1}$. By the previous lemma, $\left(Z, \tau^{\prime}\right)$ is a Tychonoff space and since $\tau^{\prime} \subseteq \tau,\left(Z, \tau^{\prime}\right)$ is pseudocompact. Furthermore, since $(Z, \tau)$ is pseudocompact, it is $G_{\delta}$-dense in $Z \cup\{p\}$ and so $\{p\}$ is not a $G_{\delta}$-set in $Z \cup\{p\}$ which implies that $\left(Z, \tau^{\prime}\right)$ does not have countable pseudocharacter, a contradiction, since it is a continuous image of $X$ which has property $P$. Thus we have proved that if $X$ has property $P$, then every continuous image of weight $\omega_{1}$ of $X$ is a first countable compact space.

Again suppose that $X$ has property $P$; we require to show that $X$ is compact. To prove this, suppose to the contrary that $\beta X \backslash X \neq \varnothing$. Let $f: \beta X \rightarrow T$ be a continuous surjection of $\beta X$ onto a space of weight at most $\omega_{1}$. Then the map $f \mid X: X \rightarrow f[X]$ is a continuous surjection of $X$ onto a space of weight at most $\omega_{1}$ and hence by the result of the previous paragraph, $f[X]$ is compact. However, $X$ is dense in $\beta X$ and so $f[X]$ is dense in $T$ and it follows that $f[X]=T$. It follows from the property $P$ of the space $X$, and $\mathrm{w}(T) \leqslant \omega_{1}$, that $\psi(T)=\chi(T)=\omega$. Since $T$ was assumed to be an arbitrary image of $\beta X$ of weight $\omega_{1}$, the space $\beta X$ also has the property $P$. However, as we saw in the second paragraph of this proof, this implies that $\beta X$ is first countable and as a consequence, $X$ is compact.

Conversely, suppose that $X$ is a perfectly normal compact space and $f: X \rightarrow Y$ is a continuous map onto a Hausdorff space $Y$. A standard argument shows that $Y$ is first countable.

It is clear from the comment at the beginning of this section, that if every continuous image of weight $\omega_{1}$ of a space $X$ has character $\omega$, then $X$ is pseudocompact. This observation allows us to formulate the following result.

Corollary 3.2 A space $X$ has the property that every continuous image of weight at most $\omega_{1}$ of $X$ has character $\omega$ if and only if $X$ is a perfectly normal compact space.

[13, Question 4.3] asks whether a space with property $P$ must have countable extent. However, if $X$ is any Tychonoff space with property $P$, then by [13, Proposition 3.3 (a)], 
every closed, discrete and $C$-embedded subset of $X$ is countable and hence $X$ is feebly Lindelöf. Thus there is a positive answer to [13, Question 4.3] in case $X$ is strongly collectionwise Hausdorff.

Recall that a space $X$ is $\omega$-bounded if the closure of every countable subset of $X$ is compact. The next theorem has a proof similar to that of Theorem 3.1; it gives a partial answer to [13, Question 4.11] and generalizes [11, Theorem 2.11] in ZFC.

Theorem 3.3 If $X$ is an $\omega$-bounded space which has countable projective tightness, then $X$ is compact and $\mathrm{t}(X)=\omega$.

Proof Suppose that $Y$ is a continuous image of $X$ of weight at most $\omega_{1}$; we claim that $Y$ is compact. To prove this, note first that if $\mathrm{w}(Y)=\omega$, then $Y$ is metrizable and hence compact. Now suppose that $\mathrm{w}(Y)=\omega_{1}$; note that $Y$ is $\omega$-bounded and we may consider $Y$ to be a subspace of $\mathbb{R}^{\omega_{1}}$. If $Y$ is not compact, then it is not realcompact and hence $Y$ is not closed in $\mathbb{R}^{\omega_{1}}$. As in Theorem 3.1, there is a point $p \in \operatorname{cl}(Y) \backslash Y$ and by identifying $p$ with a point $y \in Y$, we obtain a continuous image $Z$ of $X$ of weight $\omega_{1}$. Furthermore, since $Y$ is $\omega$-bounded, there is no countable subset of $Y$ with $p$ in its closure. However there is some, necessarily uncountable subset $A \subseteq Y$ such that $p \in \operatorname{cl}(A)$ and $y \notin \operatorname{cl}(A)$ and hence as a subset of $Z$, the set $A$ witnesses that $Z$ does not have countable tightness. This contradicts the assumption that $X$ has countable projective tightness and so $Y$ is compact.

Again suppose that the $\omega$-bounded space $X$ has countable projective tightness and suppose that $X$ is not compact, that is, $\beta X \backslash X \neq \varnothing$. Let $f: \beta X \rightarrow T$ be a continuous surjection onto a space $T$ of weight at most $\omega_{1}$. Then $f\lceil X: X \rightarrow f[X]$ has the same properties as $f$ and hence from the previous paragraph, we may conclude that $f[X]$ is compact and dense in $T$ and so $f[X]=T$. Since $X$ has countable projective tightness, it follows that $\mathrm{t}(T)=\omega$ and since $T$ is an arbitrary continuous image of $\beta X$ of weight $\omega_{1}, \beta X$ has countable projective tightness. It now follows from [11, Theorem 2.11], that $\beta X$, and hence $X$, has countable tightness. Finally, since $X$ is $\omega$-bounded, the closure in $\beta X$ of every countable subset of $X$ is contained in $X$; this combined with the countable tightness of $\beta X$ implies that $X=\beta X$, and so $X$ is compact.

The technique used in the previous two theorems can be used to give a consistent positive answer to [13, Question 4.11]. However we do not know if the next result is a theorem in ZFC. The inequality $\omega_{1}<\mathfrak{p}$ is a well known consequence of $\mathrm{MA}+\neg \mathrm{CH}$.

Theorem $3.4\left(\omega_{1}<\mathfrak{p}\right)$ If $X$ is a countably compact space of countable projective tightness, then $\mathrm{t}(X)=\mathrm{t}(\beta X)=\omega$.

Proof Let $X$ be a countably compact space of countable projective tightness and $Y$ a continuous image of $X$ with $\mathrm{w}(Y) \leqslant \omega_{1}$; note that $Y$ is countably compact. In the sequel we note only the differences between this and the proof of the previous theorem. Suppose that $q \in \operatorname{cl}(Y) \backslash Y \subseteq \mathbb{R}^{\omega_{1}}$; if $\mathrm{t}(q, Y \cup\{q\})=\omega$, there is a countable set $A \subseteq Y$ such that $q \in \operatorname{cl}(A)$. It is then a consequence of $\omega_{1}<\mathfrak{p}$ that there is an injective sequence $S$ in $A$ converging to $q$ (see [9]). However this is impossible since $S$ is closed and discrete in the countably compact space $Y$. Thus as before, we may take $q \in \operatorname{cl}(Y) \backslash Y$ such that $t(q, Y \cup\{q\})=\omega_{1}$ and so prove that $Y$ is compact. The 
proof continues as in the final paragraph of Theorem 3.3 and we conclude that $\beta X$, and hence $X$, has countable tightness.

We note that unlike Theorem 3.3, in Theorem 3.4, we cannot conclude from this proof that $X$ is compact. However, the Proper Forcing Axiom (PFA), which is consistent with $\mathrm{ZFC}$ and the existence of a supercompact cardinal, implies $\mathrm{MA}_{\omega_{1}}$ and $\mathfrak{p}=\mathfrak{c}=\omega_{2}$ (see [5]). Furthermore, PFA also implies that every compact space of countable tightness is sequential (see [2]). However, if $\beta X$ is sequential and $X$ is countably compact, then clearly, $X=\beta X$, and so $X$ is compact. On the other hand, under Jensen's $\diamond$, there exists a compact hereditarily separable space $K$ of cardinality $\mathfrak{c}$ with no non-trivial convergent sequences (see [4]). If $x \in K$, then $K \backslash\{x\}$ is a countably compact, hereditarily separable space which is not compact but which has countable projective tightness.

Question 3.5 Is it true in $\mathrm{ZFC}$ that if $X$ is a countably compact space of countable projective tightness, then $X$ has countable tightness?

Acknowledgments The authors wish to thank the anonymous referee for many helpful comments. In particular the proof of Theorem 2.2 given here is due to the referee and constitutes a very considerable simplification of our original proof.

\section{References}

1. Alas, O.T., Wilson, R.G.: The structure of the poset of regular topologies on a set. Appl. Gen. Topol. 12(1), 1-13 (2011)

2. Balogh, Z.T.: On compact Hausdorff spaces of countable tightness. Proc. Amer. Math. Soc. 105(3), 755-764 (1989)

3. Engelking, R.: General Topology. Sigma Series in Pure Mathematics, vol. 6, 2nd edn. Heldermann, Berlin (1989)

4. Fedorčuk, V.V.: A compact space having the cardinality of the continuum with no convergent sequences. Math. Proc. Cambridge Philos. Soc. 81(2), 177-181 (1977)

5. Jech, T.: Set Theory. Springer Monographs in Mathematics, 3rd edn. Springer, Berlin (2002)

6. Juhász, I.: Cardinal Functions in Topology. Mathematical Centre Tracts, vol. 34. Mathematisch Centrum, Amsterdam (1971)

7. Lutzer, D.J.: On Generalized Ordered Spaces. Dissertationes Mathematicae, vol. 89. IMPAN, Warsaw (1971)

8. Lutzer, D.J.: Ordinals and paracompactness in ordered spaces. In: Alò, R.A., Heath, R.W., Nagata, J. (eds.) TOPO 72 - General Topology and its Applications. Lecture Notes in Mathematics, vol. 378, pp. 258-266. Springer, Berlin (1974)

9. Malykhin, V.I., Shapirovskij, B.E.: Martin's axiom and properties of topological spaces. Soviet Math. Dokl. 14, 1746-1751 (1973)

10. Tkachenko, M.G., Tkachuk, V.V.: More reflections in small continuous images. Manuscript

11. Tkachuk, V.V.: Reflecting topological properties in continuous images. Cent. Eur. J. Math. 10(2), 456-465 (2012)

12. Tkachuk, V.V.: A $C_{p}$-Theory Problem Book. Problem Books in Mathematics. Springer, Cham (2014)

13. Tkachuk, V.V., Wilson, R.G.: Reflections in small continuous images of ordered spaces. Eur. J. Math. 2(2) (2016). doi:10.1007/s40879-015-0068-y 